\title{
The soft X-ray cluster-AGN spatial cross-correlation function in the ROSAT-NEP survey
}

\author{
N. Cappelluti ${ }^{1}$, H. Böhringer ${ }^{1}$, P. Schuecker ${ }^{1}$, E. Pierpaoli ${ }^{2,6}$, C. R. Mullis ${ }^{3}$, I. M. Gioia ${ }^{4}$, and J. P. Henry ${ }^{5,1}$ \\ 1 Max Planck Institute für Extraterrestrische Physik, Postfach 1312, 85741 Garching, Germany \\ e-mail: cappelluti@mpe.mpg.de \\ 2 California Institute of Technology, Mail Code 130-33, Pasadena, CA 91125, USA \\ 3 Department of Astronomy, University of Michigan, 918 Dennison, 500 Church Street, Ann Arbor, 48109-1042, USA \\ 4 Istituto di Radioastronomia INAF, via P. Gobetti 101, 40129 Bologna, Italy \\ 5 Institute for Astronomy, University of Hawai'i, 2680 Woodlawn Drive, Honolulu, HI 96822, USA \\ ${ }^{6}$ Physics and Astronomy Department, University of Southern California, Los Angeles, CA 90089-0484, USA
}

Received 27 June 2006 / Accepted 16 November 2006

\section{ABSTRACT}

\begin{abstract}
Context. X-ray surveys facilitate investigations of the environment of AGNs. Deep Chandra observations have revealed that the AGN source surface density rises near clusters of galaxies. The natural extension of this finding is the measurement of spatial clustering of AGNs around clusters and the investigation of relative biasing between active galactic nuclei and galaxies near clusters.

Aims. We aim to measure the correlation length of AGNs around clusters and the average clustering properties of a complete sample of AGNs in a dense environment.

Methods. We present the first measurement of the soft X-ray cluster-AGN cross-correlation function in redshift space using the data of the ROSAT-NEP survey. The survey covers $9 \times 9 \mathrm{deg}^{2}$ around the North Ecliptic Pole where $442 \mathrm{X}$-ray sources were detected and almost completely spectroscopically identified.

Results. We detected a $>3 \sigma$ significant clustering signal on scales $s \leq 50 h_{70}^{-1} \mathrm{Mpc}$. We performed a classical maximum-likelihood power-law fit to the data and obtained a correlation length $s_{0}=8.7_{-0.3}^{+1.2} h_{70}^{-1} \mathrm{Mpc}$ and a slope $\gamma=1.7_{-0.7}^{+0.2}(1 \sigma$ errors).

Conclusions. This is strong evidence that AGNs are good tracers of the large scale structure of the Universe. Our data were compared to the results obtained by cross-correlating X-ray clusters and galaxies. We observe, with a large uncertainty, a similar behaviour of AGN clustering around clusters similar to the clustering of galaxies around clusters.
\end{abstract}

Key words. galaxies: clusters: general - galaxies: active - X-rays: galaxies: clusters - cosmology: large-scale structure of Universe - cosmology: dark matter

\section{Introduction}

The current paradigm of galaxy formation assumes that all types of galaxies reside in dark matter (DM) haloes, and that the properties of these haloes determine to some extent the properties of the galaxies inside (White \& Rees 1978). In general, the clustering amplitudes of haloes depend on halo mass. The relation between the clustering properties of both DM haloes and galaxies (biasing) should thus tell us something about the physical processes leading to the formation and evolution of galaxies. Clusters of galaxies are the highest peaks in the global mass distribution of the Universe and should follow a direct and simple biasing scheme - mainly related to the underlying primordial Gaussian random field (Kaiser 1987). A simple means towards a better understanding of galaxy biasing is thus provided by studies of the relative biasing between galaxies and clusters of galaxies.

As a first step, previous investigations estimated two-point statistics like the auto-correlation function (Mullis et al. 2004a; Gilli et al. 2005; Basilakos et al. 2005; Yang et al. 2006). They could show that, AGNs trace the underlying cosmic large-scale structure. In addition, the large-scale structure of X-ray selected galaxy clusters could be studied in some detail (e.g. Schuecker et al. 2001), but without investigating the link between clusters and AGNs.
In the present paper, we concentrate on the study of the relative clustering between X-ray selected AGNs and galaxy clusters. Our work improves on most previous work on the largescale structure of X-ray selected AGNs in two important aspects. First, with the exception of Mullis et al. (2004a), our sample is the only one that is spectroscopically complete $(99.6 \%)$. Gilli et al. (2005) used the CDFS (35\%) and the CDFN (50\%). The Basilakos et al. (2005) sample had almost no spectroscopic redshifts. Yang et al. (2006) used the CLASXS sample (52\% complete) and the CDFN (56\% complete).

Another motivation for our work is that over the last several years, X-ray observations revealed that a significant fraction of high- $z$ clusters of galaxies show overdensities of AGNs in their outskirts (i.e. between $3 h_{70}^{-1} \mathrm{Mpc}$ and $7 h_{70}^{-1} \mathrm{Mpc}$ from the center of the cluster) (Henry et al. 1991; Cappi et al. 2001; Ruderman \& Ebeling 2005; Cappelluti et al. 2005, and references therein). These overdensities were however detected in randomly selected archive targeted observations of galaxy clusters. While these overdensities are highly significant (up to $8 \sigma$ ) when compared to cluster-free fields, the incompleteness of the samples does not allow one to draw any conclusion about the average clustering properties of AGNs around clusters. The majority of the sources making these overdensities have no spectroscopic identification and therefore any information on their 
spatial clustering is lost. More recently Branchesi et al. (2007) showed that at high- $z$ the source surface density of AGNs significantly increases, even in the central regions of the clusters. These results imply that further progress will come from studying the three dimensional spatial distribution of AGNs around clusters. A natural way to characterize this specific type of clustering is given by the three-dimensional cross-correlation of AGNs and galaxy clusters, the computation of which needs complete redshift information for all objects, which is rare in X-ray surveys.

In this respect, the ROSAT North Ecliptic Pole (NEP) survey (Henry et al. 2001, 2006; Voges et al. 2001) is one of the few $\mathrm{X}$-ray surveys covering a sufficiently large volume with an almost complete follow-up identification of AGNs and clusters (i.e. 440 sources spectroscopically identified of 442 detected). This survey thus provides a very useful basis for more precise investigations of the relative clustering properties of these two types of objects.

We organized the present paper in the following way. In Sect. 2, we describe the ROSAT-NEP survey data that we use for our investigations of the spatial distribution of X-ray selected AGNs and galaxy clusters. For the statistical analysis we estimate their cross-correlation. A useful estimator for this statistic and the mock samples needed for its determination are described in Sects. 3 and 4, respectively. The results are presented in Sect. 5, and are discussed in Sect. 6. In this paper, we assume a (concordance) Friedmann-Lemaitre Universe characterized by the Hubble constant given in units of $h_{70}=$ $H_{0} /\left(70 \mathrm{~km} \mathrm{~s}^{-1} \mathrm{Mpc}^{-1}\right)$, the normalized cosmic matter density $\Omega_{\mathrm{m}}=0.3$, and the normalized cosmological constant $\Omega_{\Lambda}=0.7$. Unless otherwise stated, errors are reported at the $1 \sigma$ confidence level.

\section{The data}

The ROSAT NEP survey covers a region of $9 \times 9 \mathrm{deg}^{2}$ around the North Ecliptic Pole $\left(17^{\mathrm{h}} 15^{\mathrm{m}}<\alpha<18^{\mathrm{h}} 45^{\mathrm{m}}, 62^{\circ}<\delta<71^{\circ}\right)$ observed with the PSPC proportional counter as part of the ROSAT All Sky Survey (Henry et al. 2001; Voges et al. 2001) with a flux limit of $2 \times 10^{-14} \mathrm{erg} \mathrm{cm}^{-2} \mathrm{~s}^{-1}$ in the $0.5-2 \mathrm{keV}$ energy band. 442 X-ray sources were detected and 440 optically identified. Spectroscopic redshift information is available for 219 AGNs and 62 clusters of galaxies. The clusters have redshifts $z \leq 0.81$ with a median of 0.18 and the AGNs have $z \leq 3.889$ with a median of 0.4 (Fig. 1). For the purpose of this work we selected all the clusters and the 185 AGNs with $z \leq 1$ (Gioia et al. 2003).

Such a dataset was used also by Mullis et al. (2004a) for the calculation of the 3-D auto correlation function of X-ray selected AGNs. Mullis and collaborators find significant clustering on scales smaller than $\sim 43 h_{70}^{-1} \mathrm{Mpc}$ with a correlation length of $\sim 10.4 h_{70}^{-1} \mathrm{Mpc}$, and a slope of the correlation best-fit power law of $\gamma=1.8$.

\section{Cluster-AGN spatial cross-correlation}

The cross-correlation function $\xi_{\mathrm{CA}}$ of clusters and AGNs is defined by the joint probability of finding, at a distance $r$, one cluster in the infinitesimal comoving volume element $\delta V_{\mathrm{C}}$ and one AGN in the comoving volume element $\delta V_{\mathrm{A}}$,

$\delta P=n_{\mathrm{C}} n_{\mathrm{A}}\left[1+\xi_{\mathrm{CA}}(r)\right] \delta V_{\mathrm{C}} \delta V_{\mathrm{A}}$,

where $n_{\mathrm{C}}$ and $n_{\mathrm{A}}$ are the mean comoving number densities of clusters and AGNs, respectively. In calculating the differential cross-correlation in redshift space we used an adapted version of the Landy-Szalay estimator (Landy \& Szalay 1993; see also e.g. Blake et al. 2006),

$\xi_{\mathrm{CA}}(s)=\frac{D_{\mathrm{C}} D_{\mathrm{A}}-R_{\mathrm{C}} D_{\mathrm{A}}-R_{\mathrm{A}} D_{\mathrm{C}}+R_{\mathrm{A}} R_{\mathrm{C}}}{R_{\mathrm{A}} R_{\mathrm{C}}}$,

where $D_{\mathrm{C}} D_{\mathrm{A}}, R_{\mathrm{C}} D_{\mathrm{A}}, R_{\mathrm{A}} D_{\mathrm{C}}$ and $R_{\mathrm{A}} R_{\mathrm{C}}$ are the normalized number of pairs in the $i$ th redshift space separation $s$ bin for the clusters data-AGN data, cluster random-AGN data, AGN random-clusters data and clusters random-AGN random samples, respectively. Using the symbols D and $\mathrm{R}$ to represent the data and random samples, respectively, and $\mathrm{C}$ and $\mathrm{A}$ to identify clusters and AGNs, respectively, the normalized pairs are expressed by

$$
\begin{gathered}
D_{\mathrm{C}} D_{\mathrm{A}}=\frac{n_{\mathrm{pair}, D_{\mathrm{A}} D_{\mathrm{C}}}\left(s_{i}\right)}{\left(N_{D_{\mathrm{A}}} \times N_{D_{\mathrm{C}}}\right)}, \quad R_{\mathrm{C}} D_{\mathrm{A}}=\frac{n_{\text {pair }, R_{\mathrm{C}} D_{\mathrm{A}}}\left(s_{i}\right)}{\left(N_{R_{\mathrm{C}}} \times N_{D_{\mathrm{A}}}\right)}, \\
R_{\mathrm{A}} D_{\mathrm{C}}=\frac{n_{\text {pair }, R_{\mathrm{A}} D_{\mathrm{C}}}\left(s_{i}\right)}{\left(N_{R_{\mathrm{A}}} \times N_{D_{\mathrm{C}}}\right)}, \quad R_{\mathrm{A}} R_{\mathrm{C}}=\frac{n_{\mathrm{pair}, R_{\mathrm{A}} R_{\mathrm{C}}}\left(s_{i}\right)}{\left(N_{R_{\mathrm{A}}} \times N_{R_{\mathrm{C}}}\right)} .
\end{gathered}
$$

Here, $N_{D_{\mathrm{A}}}, N_{D_{\mathrm{C}}}, N_{R_{\mathrm{A}}}, N_{R_{\mathrm{C}}}$ are the total numbers of AGNs and clusters in the data and in the randomly generated samples, respectively. The quantities $n_{\text {pair }}$ represent, adopting the symbolism used above, the actual number of pairs measured in the random and data samples as a function of the redshift space separation $s_{i}$. The distances were computed assuming for the position of the clusters the centroid of the X-ray emission while for AGNs the optical positions were adopted. In order to have a good signal-to-noise ratio (SNR) the data were grouped in logarithmic bins of $\Delta \log \left(s h_{70}^{-1} \mathrm{Mpc}\right)=0.15$.

\section{Random samples}

Equation (2) indicates that an accurate estimate of the distribution function of the random samples is crucial in order to obtain a reliable estimate of $\xi_{\mathrm{CA}}$. Several effects must be taken into account when generating a sample of objects in a flux limited survey. Simulated AGNs were randomly placed within the ROSAT NEP survey area. In order to reproduce the flux distribution of the real sample, we followed the method of Mullis et al. (2004a). In practice, since the cumulative AGN $\log N-\log S$ source count distribution can be described by a power law, $S=k S^{-\alpha}$, with $\alpha=1.3$, the differential probability scales as $S^{-(\alpha+1)}$. Using a transformation method (e.g. Press et al. 1986, Chap. 7) we see that the random flux above a certain X-ray flux $S_{\lim }$ is distributed as $S=S_{\lim }(1-p)^{\frac{1}{\alpha}}$, where $p$ is a random number uniformly distributed between 0 and 1 and $S_{\lim }=2 \times 10^{-14} \mathrm{erg} \mathrm{cm}^{-2} \mathrm{~s}^{-1}$, i.e. the flux limit of the NEP survey. All random AGNs with a flux lower than the flux limit map (see Fig. 4 in Henry et al. 2006) at the source position were excluded. In order to assign a redshift to these "sources" we computed the predicted redshift distribution at the position of each accepted source. Once the flux limit at the position where the source was randomly placed is known, and denoting with $\phi(L, z)$ the luminosity function, then the number of sources per redshift interval $\mathrm{d} z$ is given by

$$
N(z) \mathrm{d} z=\int_{L_{\min }}^{\infty} \int_{z}^{z+\mathrm{d} z} \phi(L, z) \mathrm{d} V(z) \mathrm{d} L \mathrm{~d} z,
$$

where $L_{\min }$ is the minimum luminosity observable at redshift $z$ with a local flux limit $S_{\lim }$ and $\mathrm{d} V(z)$ the differential comoving volume element. The $k$-correction does not play any role in the calculations since we assumed an average spectral index $\Gamma=2$ (as in Mullis et al. 2004a) for all AGNs. For the luminosity function $\phi(L, z)$ we took the luminosity-dependent density evolution 

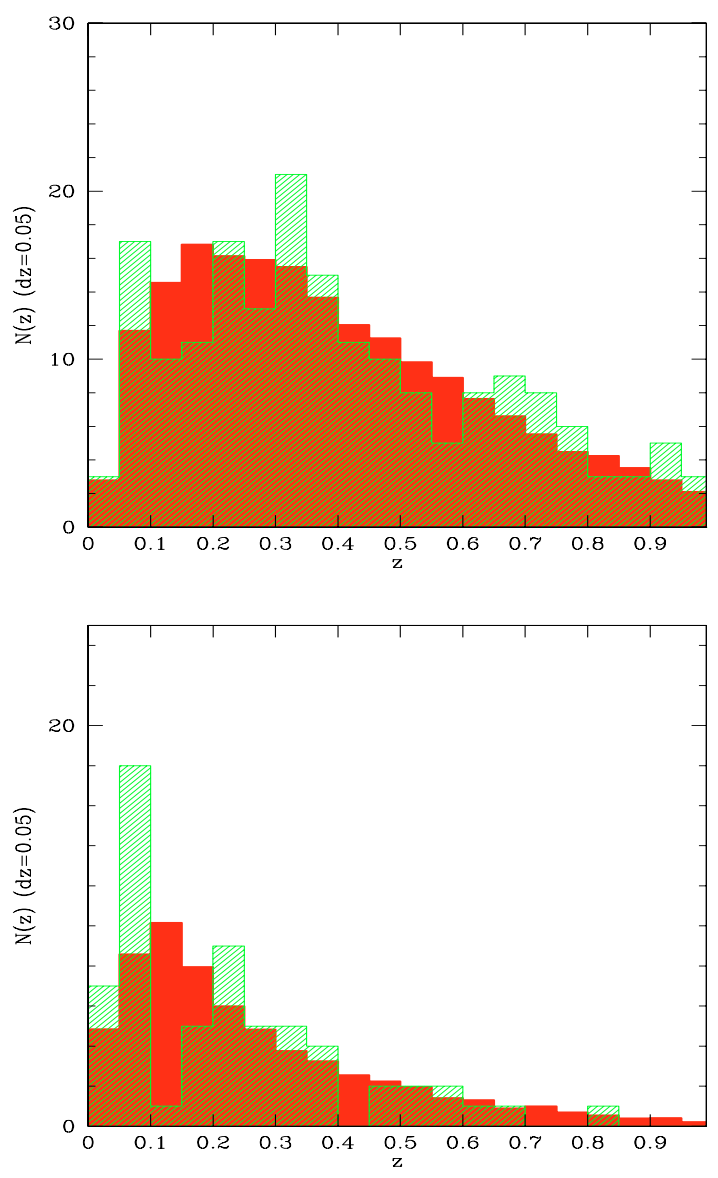

Fig. 1. Top panel: the redshift distribution of the AGNs in the NEP survey (shaded histogram) and of the randomly generated AGNs with the same selection effects (filled histogram). Bottom panel: the redshift distribution of the galaxy clusters in the NEP survey (shaded histogram) and of randomly generated clusters (filled histogram). The deviations at low $z$ between the data and the random sample are mainly caused by the NEP supercluster of galaxies (Mullis et al. 2001).

(LDDE) best fit model of Hasinger et al. (2005). A redshift was then randomly assigned to each source via Monte Carlo integration of the predicted redshift distribution. For galaxy clusters we applied the same procedure assuming a slope of the $\log N-\log S$ distribution of $\alpha=1.3$, as luminosity function an A-B evolving Schechter model (Rosati et al. 2002) with the parameters obtained by Mullis et al. (2004b) and using a sensitivity map specific for NEP clusters (Henry et al. 2006). Since for clusters of galaxies the $k$-correction is not negligible, we assigned to the random clusters an intrinsic spectrum according to a MEKAL spectral model with a plasma temperature $k T=3 \mathrm{keV}$ and a metallicity $Z=0.3 Z_{\odot}$. We also applied to $L_{\min }$ a "sizecorrection" according to the results of Henry et al. (2006) in order to compensate for the missing flux in the X-ray photometry aperture and the variation of the angular dimensions of the object with $z$. Such a procedure was repeated until we populated the survey volume with 37200 random AGNs and 12600 random clusters (i.e. 200 times more objects than in the real data sample). The redshift distribution of the random cluster and AGN samples are plotted in Fig. 1 together with the real data. To obtain a realistic estimate of the uncertainties of the cross correlations we used the bootstrap resampling technique described by e.g. Ling et al. (1986).

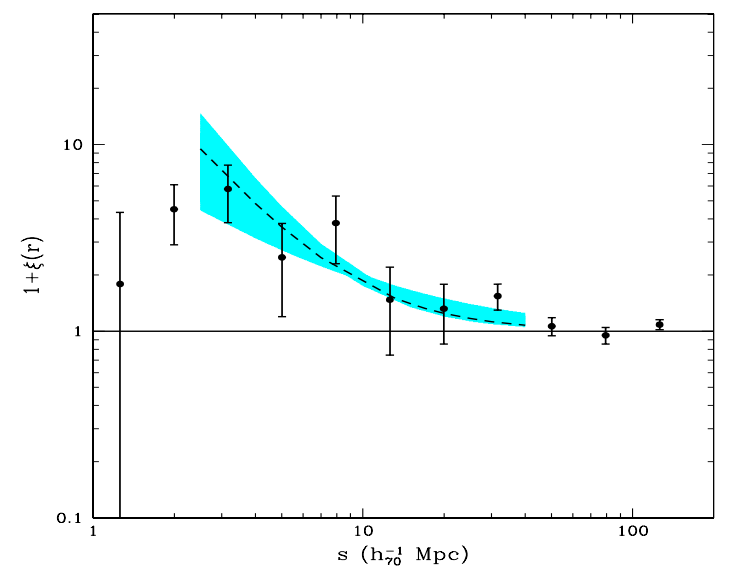

Fig. 2. The cluster-AGN soft X-ray cross correlation function plus one. The error bars are quoted at $1 \sigma$ level. The dashed line represents the best fit maximum-likelihood power-law fit $s_{0}=8.7_{-0.3}^{+1.2} h_{70}^{-1} \mathrm{Mpc}$ and $\gamma=$ $1.7_{-0.7}^{+0.2}$. The shaded region illustrates the $1 \sigma$ confidence region of the power-law fit in the distance range in which it was performed.

\section{Results}

We present the spatial cross-correlation function between clusters and AGNs in Fig. 2. A positive clustering signal is detected in the distance interval $s \leq 50 h_{70}^{-1} \mathrm{Mpc}$. In order to test the strength of the clustering we performed a canonical power-law fit,

$\xi_{\mathrm{CA}}(s)=\left(\frac{s}{s_{0}}\right)^{-\gamma}$

with $s_{0}$ and $\gamma$ as free parameters. The fit can be performed using the coarsely binned data in Fig. 2 and minimizing the $\chi^{2}$ statistics. However, this approach is extremely sensitive to the size and distribution of the bins. In order to overcome this problem we performed a standard maximum likelihood power-law fit to unbinned data. The comoving separation $s$ was parsed in very small bins so that there are either 0 or 1 data pairs for any given interval. In this regime Poisson probabilities are appropriate. In order to perform the fit, we need to find the predicted probability distribution of the cluster-AGN pairs for each value of $\gamma$ and $s_{0}$. We calculated the number of predicted pairs by replacing $\xi_{\mathrm{CA}}(s)$ in Eq. (2) with the model given by Eq. (4) and using $D_{\mathrm{C}} D_{\mathrm{A}}(s)$ (hereinafter $\left.\lambda(s) \mathrm{d} s\right)$ as variable. We can then use the separations of all the $N$ cluster-AGN pairs to form a likelihood function. This is defined as the product of the probabilities of having exactly one pair in the interval $\mathrm{d} s$ at each separation $s_{i}$ of the $N$ pairs times the probability of having no pairs in all the other differential intervals. This holds for all $s$ in a chosen range $\left(s_{a}-s_{b}\right)$, in our case where $\xi_{\mathrm{CA}}(s)$ can be reasonably represented by a power law. Assuming Poisson probabilities we thus obtain the likelihood

$\mathcal{L}=\prod_{i}^{N} \lambda\left(s_{i}\right) \mathrm{d} s \exp \sum_{j \neq i} \lambda\left(s_{j}\right) \mathrm{d} s$,

where $\lambda\left(s_{i}\right) \mathrm{d} s$ is the expected number of pairs in the interval $\mathrm{d} s$, and the index $j$ runs over all the elements $\mathrm{d} s$ that do not contain pairs. We then define the usual quantity $S=-2 \ln \mathcal{L}$ and drop the terms independent of model parameters (see e.g. Schuecker \& Boehringer 1998; Croft et al. 1997) leading to

$S=2 \int_{s_{a}}^{s_{b}} \lambda(s) \mathrm{d} s-2 \sum_{i}^{N} \ln \left[\lambda\left(s_{i}\right)\right]$. 


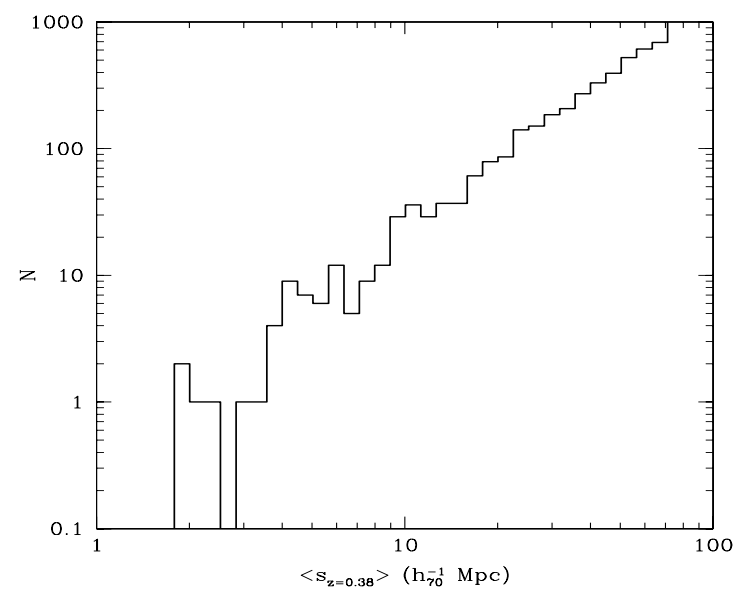

Fig. 3. The distribution of the angular radial separations translated into redshift space distances at $\langle z\rangle=0.38$.

In order to check in which range of separations we can conduct our analysis, we transformed the angular separations between clusters and AGNs into an average redshift space separation. We assumed that all the sources were at $\langle z\rangle=0.38$ (i.e. the median redshift of the cluster and AGN sample). The result is plotted in Fig. 3. As one can see there are no real pairs with separation $<2 h_{70}^{-1} \mathrm{Mpc}$. For this reason we decided not to consider points at a separation lower than $2.5 h_{70}^{-1} \mathrm{Mpc}$. These points in the cross-correlation function are mainly introduced by the parameters $R_{\mathrm{C}} D_{\mathrm{A}}, R_{\mathrm{A}} D_{\mathrm{C}}$ in Eq. (2) which could have smaller separations than the real data since the random sample includes neither the extended emission of the clusters nor the broadening due to the PSF of pointlike sources. This prevents us also from overestimating the correlation length introduced by the amplification bias (see e.g. Vikhlinin \& Forman 1995). For this reason and since on scales larger than $50 h_{70}^{-1} \mathrm{Mpc}$ there is no evidence of signal, the fit was performed over the distance range 2.5-50 $h_{70}^{-1} \mathrm{Mpc}$. In Fig. 4 we show the results of the maximum-likelihood power-law fit to $\xi_{\mathrm{CA}}(s)$ for the ROSAT NEP survey. The 1,2 and $3 \sigma$ were obtained at $\Delta S$ levels of 2.3 , 6.2 and 11.8 from the minimum value of $S$. The best fit parameters obtained are $s_{0}=8.7_{-0.3}^{+1.2} h_{70}^{-1} \mathrm{Mpc}$ and $\gamma=1.7_{-0.7}^{+0.2}$ where the uncertainty is at the $1 \sigma$ confidence level. With $\gamma$ fixed to 1.8 (i.e. a typical value found in galaxy-galaxy correlation functions) we find $s_{0} \sim 8.5 h_{70}^{-1} \mathrm{Mpc}$; a similar value was obtained by extending the fitting region to $60 h_{70}^{-1} \mathrm{Mpc}$ and restricting it to the 2.5-40 $h_{70}^{-1} \mathrm{Mpc}$.

The integral constraint (Peebles 1980), which is a systematic shift in correlation functions introduced by the limited volume observed, was computed following the prescription of Roche et al. (1993). This can be obtained numerically with a fit by assuming that the correlation function is represented by a power law of fixed index (here we used $\gamma=1.7$ ) on all scales sampled by the survey. The underestimate of $\xi_{\mathrm{CA}}(s)$ due to the integral constraint is found to be of the order of $2 \%$.

In order to determine the stability of these results the procedure was repeated first by separating the field in two subfields twice, the North and South, and West and East parts of the survey. The fluctuations due to sample variance are found to be smaller than the typical amplitude of the uncertainties. A similar result is obtained by recomputing the $\xi_{\mathrm{CA}}(s)$ for clusters with $z \leq 0.18$ (i.e. the cluster median redshift) and $z>0.18$. The dependence of $\xi_{\mathrm{CA}}(s)$ on the cluster X-ray luminosity $\left(L_{\mathrm{X}}\right)$ was evaluated, dividing the cluster sample into two subsamples with

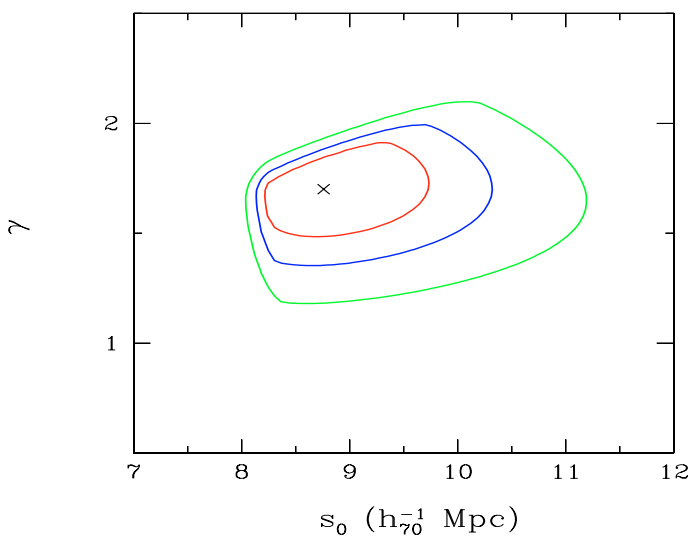

Fig. 4. The $1 \sigma, 2 \sigma$ and $3 \sigma$ confidence contours in the $s_{0}, \gamma$ space for the power-law fit to $\xi_{\mathrm{CA}}(s)$ for two interesting parameters. The cross represents the best fit values.

$L_{\mathrm{X}} \leq($ or $\geq) 3.8 \times 10^{43} h_{70}^{-2} \mathrm{erg} \mathrm{s}^{-1}$. Though no significant $L_{\mathrm{X}}$ dependent behaviour was detected, we cannot yet conclude that there is no luminosity or redshift dependent cross-correlation length because of the statistics of the sub-samples. The stability of the result was also checked by fitting the data with the likelihood estimator used by Mullis et al. (2004a), returning no significant deviations from our results at a $1-2 \sigma$ level.

\section{Discussion}

We presented here the first direct evidence of spatial clustering of soft X-ray selected AGNs around X-ray selected clusters of galaxies. Indirect evidence was presented by Henry et al. (1991), Cappi et al. (2001), Cappelluti et al. (2005) (and references therein). These authors found significant X-ray point source overdensities (about a factor 2) around distant clusters of galaxies when compared to cluster-free fields. If the overdensities were at the cluster redshift they would arise at scales smaller than $\sim 7 h_{70}^{-1} \mathrm{Mpc}$. Since the correlation function is proportional to $\left(\frac{\delta \rho}{\rho}\right)^{2}$, a $\xi_{\mathrm{CA}}=1$ implies an overdensity of a factor of 2 with respect to a randomly distributed field. We can conclude that, since the correlation length found in this work reflects the scale of the overdensities known up to now, we observe a physical overdensity (of at least a factor of 2) of AGNs around clusters between 2 and $\sim 8 h_{70}^{-1} \mathrm{Mpc}$ from the center of the clusters.

Because of the shallowness of the NEP survey, the AGN surface density (i.e. $<30 \mathrm{deg}^{2}$ in the central region) does not allow detection of such a correlation via overdensity analysis since it would be dominated by small number statistics. From our results we expect to detect AGN overdensities on scales $<7-8 h_{70}^{-1} \mathrm{Mpc}$ from the center of clusters. At $\langle z\rangle \sim 0.18$ (i.e. the median $z$ of the cluster sample of the NEP survey) these overdensities arise on scales of $\sim 0.6 \mathrm{deg}^{-2}$ which are easily resolved by the NEP survey. However, to significantly detect these overdensity on single clusters, a large number of sources is necessary to disentangle real overdensities from shot noise. This problem could be easily resolved by high angular resolution telescopes like Chandra and partially by XMM-Newton. In this direction deep and wide ${ }^{1}$ Chandra and XMM-Newton surveys like COSMOS, which will

1 The expected fluxes limit for the C-COSMOS and XMMCOSMOS survey are $1-2 \times 10^{-16} \mathrm{erg} \mathrm{cm}^{-2} \mathrm{~s}^{-1}$ and $\sim 6 \times$ $10^{-16} \mathrm{erg} \mathrm{cm}^{-2} \mathrm{~s}^{-1}$, respectively. These surveys will cover $0.9 \mathrm{deg}^{2}$ and $2.1 \mathrm{deg}^{2}$, respectively. 
return an AGN surface density of up to $\sim 2700 \mathrm{deg}^{-2}$, would allow seeing in a $0.015 \mathrm{deg}^{2}$ region (i.e. the size of an ACIS-I chip) a population of at least 40 AGNs belonging to the cluster environment (i.e. assuming an average overdensity of a factor 2 , we expect $\sim 40$ sources belonging to the cluster and $\sim 40$ to the background). As an example, at the limiting flux of the $\mathrm{C}$ COSMOS survey, the AGN population of a cluster at $z=1$ would be observed with a $0.5-2 \mathrm{keV}$ limiting luminosity $L_{\min } \sim$ $10^{42} \mathrm{erg} \mathrm{s}^{-1}$. At $z=1$ the size of an ACIS chip (i.e. 8 arcmin) corresponds to a linear dimension of $\sim 4 h_{70}^{-1} \mathrm{Mpc}$. Having 40 AGNs in a sphere with this radius corresponds to a space density of AGN with $L_{\mathrm{X}}>L_{\text {min }}$ of $\sim 0.15 h_{70}^{3} \mathrm{Mpc}^{-3}$. According to the result presented here, clusters of galaxies could be detected by AGN overdensities (rather than galaxy overdensities) if the depth of the survey provides an AGN surface density sufficient to overcome the Poisson noise on the AGN number. In general, in order to understand the galaxy evolution in dense environments the measure of cross correlation between clusters and different kind of galaxies is an important tool. We already know that infrared dusty galaxies avoid dense environments, therefore showing a large cross-correlation length and a weak clustering signal in the small separations region (Sánchez et al. 2005). We also know that blue galaxies avoid low- $z$ rich clusters cores (Butcher \& Oemler 1984). It is therefore important to compare the clusterAGN cross correlation length to that of clusters and different galaxy types. Mo et al. (1993) computed the cross-correlation function of Abell clusters and QDOT IRAS galaxies. They found an average correlation length and a slope in agreement with the results presented here. Moreover Lilje \& Efstathiou (1988) showed that the cross-correlation function of Abell clusters with Lick galaxies is positive on scales $\leq 29 h_{70}^{-1} \mathrm{Mpc}$ with a slope $\gamma \sim 2.2$ and a correlation length of $\sim 12.6 h_{70}^{-1} \mathrm{Mpc}$. These results are also in agreement within $1 \sigma$ with our findings on AGNs. These first comparisons suggest that AGNs are clustered around galaxy clusters just like galaxies. As a final check we compared our $\xi_{\mathrm{CA}}$ to the X-ray cluster-galaxy cross-correlation function (hereinafter CGCCF) computed by Sánchez et al. (2005). They used the X-ray selected clusters of the REFLEX survey (Böhringer et al. 2002) and the galaxies from the APM survey (Maddox et al. 1990) limited to $b_{j}=20.5 \mathrm{mag}$. They found that the CGCCF behaves like a broken power-law with a cut-off distance of $\sim 2 h_{70}^{-1} \mathrm{Mpc}$ with a steeper slope at small distances. We can define the following approximate biasing relations:

$\xi_{\mathrm{CA}}(s)=b_{\mathrm{C}} b_{\mathrm{A}} \xi_{\rho}(s), \quad \xi_{\mathrm{CG}}(s)=b_{\mathrm{C}} b_{\mathrm{G}} \xi_{\rho}(s)$.

Here $\xi_{\rho}(s)$ is the autocorrelation of matter, $b_{\mathrm{G}}, b_{\mathrm{A}}$ and $b_{\mathrm{C}}$ are the bias factors relative to galaxies, AGNs and clusters, respectively. By dividing the two equations we can then derive $\frac{b_{\mathrm{A}}}{b_{\mathrm{G}}}(s)$. In order to perform this operation, several effects must be taken in account.

- The bias of REFLEX clusters could be slightly different from that of NEP clusters since they are differently distributed in redshift and the surveys have different limiting fluxes and X-ray luminosity distributions. Schuecker et al. (2001) showed with the power spectrum that even having a large sample of clusters as REFLEX the error on the bias determination is still high. Since in the NEP survey we expect an even higher uncertainty, it is a reasonable approximation to consider the ratio $\frac{b_{\mathrm{NEP}}}{b_{\mathrm{REF}}}$ consistent with 1 .

- Sánchez et al. (2005) computed $\xi_{\mathrm{CG}}$ in real space while we work in redshift space. In order to evaluate the effect of redshift space distortion on $\xi_{\mathrm{CG}}$ we used the results of

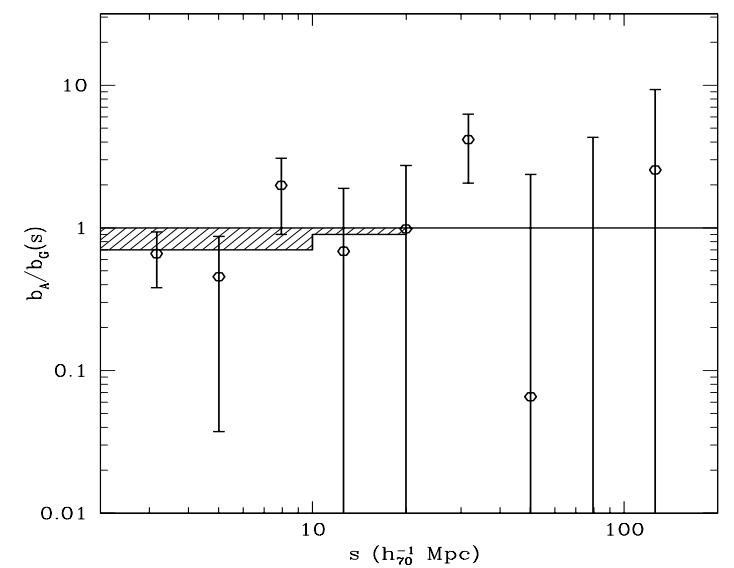

Fig. 5. The ratio between the observed ROSAT NEP $\xi_{\mathrm{CA}}(s)$ and the best fit $\xi_{\mathrm{CG}}(r)$ obtained by Sánchez et al. (2005). Errors are quoted at the $1 \sigma$ level. The shaded region shows the expected level of $\frac{b_{\mathrm{A}}}{b_{\mathrm{G}}}(s)=1$ if the cross-correlation functions were compared in the same space.

Croft et al. (1999). Their Figs. 3 and 5 show $\xi_{\mathrm{CG}}$ computed both in redshift and real space. From that work we estimate that our relative bias is affected by a $\sim 30 \%$ overestimate below $10 h_{70}^{-1} \mathrm{Mpc}$ and of $\sim 10 \%$ between $10 h_{70}^{-1} \mathrm{Mpc}$ and $20 h_{70}^{-1} \mathrm{Mpc}$. Their work also indicates that $\xi_{\mathrm{CG}}$ does not depend on the richness of the clusters used in the calculation and that the correction for the scale independent biasing on large scales (Kaiser 1987) can be neglected when compared to the size of our uncertainties.

The ratio $\frac{b_{\mathrm{A}}}{b_{\mathrm{G}}}(s)$ is plotted in Fig. 5 as a function of the distance from the center of the cluster. The shaded region shows the value of our measurement that implies that $\frac{b_{\mathrm{A}}}{b_{\mathrm{G}}}(s)=1$ when taking into account the difference between real and redshift space measurements discussed in the previous paragraph. The ratio is consistent with 1 on almost all scales. We cannot exclude, within the errors, different values of the relative bias. Our data suggest an average relative bias consistent with unity but allow an upper limit of $\sim 6$ (at $1 \sigma$ ) at separations $s<50 h_{70}^{-1} \mathrm{Mpc}$. For separations $s>10 h_{70}^{-1} \mathrm{Mpc}$ no lower limits larger than zero can be given. On larger scales the error increases thus it is difficult to draw any conclusion. At large separations the power-law shape of $\xi_{\mathrm{CG}}$ becomes uncertain, this makes a comparison of our data with those of Sánchez et al. (2005) less meaningful. We cannot exclude a significant antibiasing of AGNs when compared to galaxies, especially at low separations. Though the amplitude of the uncertainties of our data still allows a fluctuation in the relative biasing of more than a factor of 2 , we can conclude with a precision of $1 \sigma$ that the probability for a galaxy to become an AGN is constant in the range of separations sampled in this work and that AGNs can be considered as a tracer of the dark matter distribution as are galaxies. New deep and wide field surveys such as XMM-COSMOS (Hasinger et al. 2006; Cappelluti et al. 2007) and Chandra-COSMOS (P.I.: Martin Elvis) with their spectroscopically identified $>2000$ AGNs and $\sim 100$ clusters will improve the precision of this work and will allow us to investigate the behaviour of AGNs in the inner regions of the clusters. In this region the observations suggest that the AGN source density is affected by other physical effects such as merging (Ruderman \& Ebeling 2005; Branchesi et al. 2006) or ram pressure phenomena. 


\section{Summary}

We derived for the first time the soft X-ray spatial crosscorrelation function between clusters and AGNs using the data of the ROSAT NEP survey. A strong clustering signal was detected on scale $s<50 h_{70}^{-1} \mathrm{Mpc}$. The best power-law fit parameters are $s_{0}=8.7_{-0.3}^{+1.2} h_{70}^{-1} \mathrm{Mpc}$ and $\gamma=1.7_{-0.7}^{+0.2}$. In this work we observed that the source density of AGNs is higher near clusters than in the field. This result confirms earlier findings of overdensities of AGNs around clusters reported by many authors and improves the evidence connecting the overdensities to the large scale structure of the Universe. We also derived the relative bias between AGNs and galaxies which is consistent with one on almost all scales investigated here. This result still allows, within the errors, a factor of 2 fluctuation. New wide field surveys (such as XMM-COSMOS) performed with the new generation X-ray telescopes will be useful to enlarge the statistics, to better understand the physics of AGNs in clusters and to extend the analysis to the inner regions of clusters.

Acknowledgements. N.C. thanks Günther Hasinger for useful discussions, and Marica Branchesi for advanced communication of her results. E.P. is an ADVANCE fellow (NSF grant AST-0340648), also supported by NASA grant NAG5-11489. J.P.H. thanks the Alexander von Humboldt Foundation for support to visit the MPE. We also thank the anonymous referee for her/his useful comments and suggestions.

In memory of Peter Schuecker.

\section{References}

Basilakos, S., Plionis, M., Georgakakis, A., \& Georgantopoulos, I. 2005, MNRAS, 356, 183

Blake, C., Pope, A., Scott, D., \& Mobasher, B. 2006, MNRAS, 368, 732

Böhringer, H., Collins, C. A., Guzzo, L., et al. 2002, ApJ, 566, 93

Branchesi, M., Gioia, I. M., Fanti, C., Fanti, R., \& Perley, R. 2006, A\&A, 446, 97
Branchesi, M., Gioia, I. M., Fanti, C., Fanti, R., \& Cappelluti, N. 2007, A\&A, 462,449

Butcher, H., \& Oemler, A., Jr. 1984, ApJ, 285, 426

Cappelluti, N., Cappi, M., Dadina, M., et al. 2005, A\&A, 430, 39

Cappelluti, N., et al. 2007, ApJS, [astro-ph/0701196]

Cappi, M., Mazzotta, P., Elvis, M., et al. 2001, ApJ, 548, 624

Croft, R. A. C., Dalton, G. B., Efstathiou, G., Sutherland, W. J., \& Maddox, S. J. 1997, MNRAS, 291, 305

Croft, R. A. C., Dalton, G. B., \& Efstathiou, G. 1999, MNRAS, 305, 547

Hasinger, G., Miyaji, T., \& Schmidt, M. 2005, A\&A, 441, 417

Hasinger, G., et al. 2006, ApJS, [astro-ph/0612311]

Gilli, R., Daddi, E., Zamorani, G., et al. 2005, A\&A, 430, 811

Gioia, I. M., Henry, J. P., Mullis, C. R., et al. 2003, ApJS, 149, 29

Henry, J. P., \& Briel, U. G. 1991, A\&A, 246, L14

Henry, J. P., Gioia, I. M., Mullis, C. R., et al. 2001, ApJ, 553, L109

Henry, J. P., Mullis, C. R., Voges, W., et al. 2006, ApJS, 162, 304

Kaiser, N. 1987, MNRAS, 227, 1

Landy, S. D., \& Szalay, A. S. 1993, ApJ, 412, 64

Lilje, P. B., \& Efstathiou, G. 1988, MNRAS, 231, 635

Ling, E. N., Barrow, J. D., \& Frenk, C. S. 1986, MNRAS, 223, 21P

Maddox, S. J., Efstathiou, G., Sutherland, W. J., \& Loveday, J. 1990, MNRAS, $242,43 \mathrm{P}$

Mo, H. J., Peacock, J. A., \& Xia, X. Y. 1993, MNRAS, 260, 121

Mullis, C. R., Henry, J. P., Gioia, I. M., et al. 2001, ApJ, 553, L115

Mullis, C. R., Henry, J. P., Gioia, I. M., et al. 2004a, ApJ, 617, 192

Mullis, C. R., Vikhlinin, A., Henry, J. P., et al. 2004b, ApJ, 607, 175

Peebles, P. J. E. 1980, The large-scale structure of the universe (Princeton, N.J.: Princeton University Press)

Press, W. H., Teukolsky, S. A., Vetterling, W. T., \& Flannery, B. P. 1986, Numerical Recipes in Fortran (Cambrige University Press)

Roche, N., Shanks, T., Metcalfe, N., \& Fong, R. 1993, MNRAS, 263, 360

Rosati, P., Borgani, S., \& Norman, C. 2002, ARA\&A, 40, 539

Ruderman, J. T., \& Ebeling, H. 2005, ApJ, 623, L81

Sánchez, A. G., Lambas, D. G., Böhringer, H., \& Schuecker, P. 2005, MNRAS, 362,1225

Schuecker, P., \& Boehringer, H. 1998, A\&A, 339, 315

Schuecker, P., Böhringer, H., Guzzo, L., et al. 2001, A\&A, 368, 86

Vikhlinin, A., \& Forman, W. 1995, ApJ, 455, L109

Voges, W., Henry, J. P., Briel, U. G., et al. 2001, ApJ, 553, L119

White, S. D. M., \& Rees, M. J. 1978, MNRAS, 183, 341

Yang, Y., Mushotzky, R. F., Barger, A. J., \& Cowie, L. L. 2006, ApJ, 645, 68 